\title{
Electronic Transport at Low Temperatures: Diagrammatic Approach
}

\author{
D. Belitz ${ }^{\mathrm{a}, 1}$, T.R. Kirkpatrick ${ }^{\mathrm{b}}$ \\ ${ }^{a}$ Department of Physics and Institute of Theoretical Science, University of Oregon, Eugene, OR 97403, USA \\ b Institute for Physical Science and Technology and Department of Physics, University of Maryland, College Park, MD \\ 20742, USA
}

\begin{abstract}
We prove that a diagrammatic evaluation of the Kubo formula for the electronic transport conductivity due the exchange of bosonic excitations, in the usual conserving ladder approximation, yields a result consistent with the Boltzmann equation. In particular, we show that an uncontrolled approximation that has been used to solve the integral equation for the vertex function is unnecessary. An exact solution of the integral equation yields the same asymptotic low-temperature behavior as the approximate one, albeit with a different prefactor, and agrees with the temperature dependence of the Boltzmann solution. Examples considered are electron scattering from acoustic phonons, and from helimagnons in helimagnets.
\end{abstract}

Key words: electronic transport theory, electron-phonon scattering, helical spin waves PACS: 72.10.Bg, 72.10.Di, 75.30.Ds

\section{Introduction}

The earliest systematic treatment of electronic transport in solids was obtained by using the Boltzmann equation. The latter is known to become exact in the limit of vanishing scatterer density. This implies that it becomes exact in the limit of low temperatures for scattering processes that involve excitations, such as phonons, that get frozen out in the limit of zero temperature. For most problems, the Boltzmann equation cannot be solved in closed form, but systematic schemes of successive approximations exist; for instance, the method of variational solutions [1]. Usually, the lowest order variational solution yields the exact temperature dependence of the transport coefficients; one of the best known examples is the

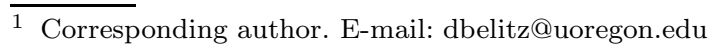

Bloch-Grüneisen law for the electrical resistivity $\rho$ due to acoustic phonons, which states that the leading temperature dependence of $\rho$ is given by $\rho \propto T^{5}[1]$.

A different approach to transport phenomena uses the Kubo formula [2], which expresses the transport coefficients in terms of equilibrium time correlation functions that can be analyzed by means of manybody perturbation theory. For the electrical conductivity, the relevant correlation function is the currentcurrent correlation. It is known that an analysis that respects the conservation of charge requires a consistent treatment of self-energy and vertex-correction diagrams. As a result, the transport relaxation rate is different from the single-particle relaxation rate, and the former is much harder to calculate than the latter. The problem can be cast in the form of an integral equation for the energy-dependent transport relaxation rate $1 / \tau_{\operatorname{tr}}(\epsilon)$ that is not easy to solve [3]. An 
approximation that ignores the energy dependence of $1 / \tau_{\operatorname{tr}}(\epsilon)[4]$ reduces the integral equation to an algebraic equation and leads to a result that reproduces the Bloch-Grüneisen result for the electrical transport rate due to electron-phonon scattering. This is in contrast to the single-particle rate due to phonons, which is proportional to $T^{3}$. However, because of the known energy dependence of the electronic self energy, which can be quite pronounced, this approximation has been questioned, and even the very validity of the $T^{5}$ law as derived from many-body perturbation theory has been called into question[4]. While such considerations are not consistent with what is known from the Boltzmann equation, and thus should cast more doubt on the Kubo formula or its evaluation then on the BlochGrüneisen law, they have led to a popular notion that the $T^{5}$ law is not firmly established. In any case, reducing the integral equation to an algebraic one is indeed an uncontrolled approximation that can be justified only by reference to the Boltzmann equation, not within the diagrammatic formalism itself.

Scattering by excitations other than phonons leads to analogous questions. For instance, in the ordered phase of helical magnets such as MnSi the Goldstone modes associated with the helical order - helimagnons - lead to a single-particle relaxation rate proportional to $T^{3 / 2}$, and the approximation mentioned above suggests a transport rate proportional to $T^{5 / 2}$ [5]. While this result is extremely plausible by analogy to the electron-phonon problem, and can be checked by solving the Boltzmann equation, it would nevertheless be very valuable to ascertain it directly within the manybody formalism. The purpose of this paper is to provide this missing link: We prove that the integral equation for the transport relaxation rate has a solution that is proportional to $T^{5}$ for the electron-phonon problem, and to $T^{5 / 2}$ for the electron-helimagnon problem.

\section{Integral equation for the transport relaxation rate}

Consider electrons scattered by excitations that lead to an effective scattering potential with a spectrum $V^{\prime \prime}(\mathbf{p}, u)$, where $\mathbf{p}$ and $u$ denote the wave vector and the frequency, respectively. Standard developments [4] lead to the following expression for the electrical con- ductivity $\sigma$ :

$$
\sigma=\frac{n_{\mathrm{e}} e^{2}}{2 m_{\mathrm{e}}} \int_{-\infty}^{\infty} d \epsilon\left(\frac{-\partial f}{\partial \epsilon}\right) \frac{\Lambda(\epsilon)}{\Gamma_{0}(\epsilon)},
$$

with $n_{\mathrm{e}}$ the electron density and $m_{\mathrm{e}}$ the electron mass. If one defines

$$
\begin{aligned}
& \bar{V}_{m}^{\prime \prime}(u)=\frac{1}{2 k_{\mathrm{F}}^{2}} \int_{0}^{2 k_{\mathrm{F}}} d p p\left(\frac{p^{2}}{2 k_{\mathrm{F}}^{2}}\right)^{m} V^{\prime \prime}(\mathbf{p}, u) \\
&(m=0,1),
\end{aligned}
$$

then the energy-dependent single-particle relaxation rate $\Gamma_{0}$ is given by

$$
\Gamma_{0}(\epsilon)=-N_{\mathrm{F}} \int_{-\infty}^{\infty} d u\left[n\left(\frac{u}{T}\right)+f\left(\frac{u+\epsilon}{T}\right)\right] \bar{V}_{0}^{\prime \prime}(u),
$$

with $N_{\mathrm{F}}$ the electronic density of states per spin at the Fermi surface, and $n(x)$ and $f(x)$ the Bose and Fermi distribution functions, respectively. For a given $\bar{V}_{0}^{\prime \prime}$, $\Gamma_{0}$ is an explicitly known function of $T$ and $\epsilon / T$. The vertex part $\Lambda$ is given as the solution of the integral equation

$$
\begin{aligned}
\Lambda(\epsilon)=1-N_{\mathrm{F}} \int_{-\infty}^{\infty} d u\left[n\left(\frac{u}{T}\right)+f\left(\frac{u+\epsilon}{T}\right)\right] \\
\quad \times\left[\bar{V}_{0}^{\prime \prime}(u)-\bar{V}_{1}^{\prime \prime}(u)\right] \frac{\Lambda(\epsilon+u)}{\Gamma_{0}(\epsilon+u)} .
\end{aligned}
$$

The transport relaxation rate $\Gamma_{\operatorname{tr}}(\epsilon) \equiv \Lambda(\epsilon) / \Gamma_{0}(\epsilon)$ is the solution of

$$
\frac{1}{\Gamma_{\operatorname{tr}}(\epsilon)}=\frac{1}{\Gamma_{0}(\epsilon)}+\int_{-\infty}^{\infty} d u \frac{K_{0}(\epsilon, u)-K_{1}(\epsilon, u)}{\Gamma_{\operatorname{tr}}(u)},
$$

where

$K_{0,1}(\epsilon, u)=-N_{\mathrm{F}}\left[n\left(\frac{u-\epsilon}{T}\right)+f\left(\frac{u}{T}\right)\right] \frac{\bar{V}_{0,1}^{\prime \prime}(u-\epsilon)}{\Gamma_{0}(\epsilon)}$.

We will be interested in problems where the frequency in the spectrum $V^{\prime \prime}(\mathbf{p}, u)$ scales as a power of the wave number, so that $\bar{V}_{1}^{\prime \prime}(u) / \bar{V}_{0}^{\prime \prime}(u) \rightarrow 0$ for $u \rightarrow 0$. Since both the frequency $u$ and the energy $\epsilon$ scale as the temperature, this implies that, effectively, $K_{1} / K_{0} \rightarrow 0$ for $T \rightarrow 0$. This observation will be important later.

\section{Analysis of the integral equation}

From Sec. 2 we see that we need to analyze the integral equation 


$$
\varphi(\epsilon)=\frac{1}{\Gamma_{0}(\epsilon)}+\int_{-\infty}^{\infty} d u\left[K_{0}(\epsilon, u)-K_{1}(\epsilon, u)\right] \varphi(u)
$$

where the first part of the kernel has the property

$$
\int_{-\infty}^{\infty} d u K_{0}(\epsilon, u)=1
$$

which follows from Eq. (3). Equation (7a) is a Fredholm equation of the second kind, and in what follows we will use the known properties of this class of integral equations [6]. We will refer to equations in Chapter 1 of Ref. [6] as ( $\mathrm{S} x \mathrm{x})$, and to theorems by section number, (S Sec.n Theorem m).

\subsection{The homogeneous equation with kernel $K_{0}$}

We first consider the homogeneous integral equation

$$
\varphi(\epsilon)=\lambda \int_{-\infty}^{\infty} d u K_{0}(\epsilon, u) \varphi(u) .
$$

It follows from Eq. (7b) that $\lambda=\lambda_{0}=1$ is an eigenvalue of the kernel $K_{0}$ with an eigenfunction $\varphi_{0}(\epsilon) \equiv 1$, (S 34,35). Note that it also follows from Eq. (7b) that $\int_{-\infty}^{\infty} d \epsilon d u K_{0}(\epsilon, u)=\infty$. This means that the sufficient condition (S 40ff) for an iterative solution of Eq. (7a) is badly violated, which suggests that iteration will not converge. This in turn suggests that the behavior of $\Gamma_{\mathrm{tr}}$ will be qualitatively different from that of $\Gamma_{0}$.

It is also useful to consider the adjoint of Eq. (8),

$$
\psi(\epsilon)=\lambda \int_{-\infty}^{\infty} d u K_{0}(u, \epsilon) \psi(u) .
$$

From (S Sec.10 Theorem 9) it follows that for $\lambda=\lambda_{0}=$ 1 Eq. (9) has a nonvanishing solution; i.e., there exists a nonvanishing function $\psi_{0}(\epsilon)$ that obeys

$$
\psi_{0}(u)=\int_{-\infty}^{\infty} d \epsilon \psi_{0}(\epsilon) K_{0}(\epsilon, u)
$$

\subsection{The inhomogeneous equation with kernel $K_{0}$}

We next consider the inhomogeneous equation

$$
\varphi(\epsilon)=\frac{1}{\Gamma_{0}(\epsilon)}+\lambda \int_{-\infty}^{\infty} d u K_{0}(\epsilon, u) \varphi(u) .
$$

(S Sec.10 Theorem 10) says that a necessary and sufficient condition for the existence of a solution of Eq. (11) is

$$
\int_{-\infty}^{\infty} d \epsilon \frac{1}{\Gamma_{0}(\epsilon)} \psi(\epsilon)=0
$$

for every solution $\psi(\epsilon)$ of Eq. (9). An explicit check of this condition would require to explicitly find the solutions of Eq. (9), i.e., the left eigenfunctions of the kernel $K_{0}$, which is difficult. However, we will see in what follows that one can construct perturbative solutions of Eq. (7a) that do not exist as $K_{1} \rightarrow 0$, which implies that Eq. (12) cannot hold for all solutions of Eq. (9).

\subsection{A perturbative eigenvalue of the full kernel}

Now we consider the following generalization of Eq. (7a),

$$
\varphi(\epsilon)=\frac{1}{\Gamma_{0}(\epsilon)}+\lambda \int_{-\infty}^{\infty} d u\left[K_{0}(\epsilon, u)-\alpha K_{1}(\epsilon, u)\right] \varphi(u),
$$

which reduces to Eq. (7a) for $\lambda=\alpha=1$. Our goal is to use $\alpha$ as a small parameter. It follows from the observation at the end of Sec. 2 that working to leading order in $\alpha$ and then setting $\alpha=1$ will yield a result that is valid to leading order in $T$ as $T \rightarrow 0$.

We know from Sec. 3.1 that for $\alpha=0, \lambda=\lambda_{0}=1$ is an eigenvalue of the kernel with eigenfunction $\varphi_{0}(\epsilon) \equiv$ 1. For the generalization of the eigenproblem of Sec. 3.1 to the full kernel $K=K_{0}-\alpha K_{1}$,

$$
\varphi_{0}(\epsilon)=\lambda_{0} \int_{-\infty}^{\infty} d u K(\epsilon, u) \varphi_{0}(u)
$$

this suggests a perturbative expansion

$$
\lambda_{0}=\lambda_{0}^{(0)}+\alpha \lambda_{0}^{(1)}+O\left(\alpha^{2}\right)
$$

with $\lambda_{0}^{(0)}=1$, and

$$
\varphi_{0}(\epsilon)=\varphi_{0}^{(0)}(\epsilon)+\alpha \varphi_{0}^{(1)}(\epsilon)+O\left(\alpha^{2}\right),
$$

with $\varphi_{0}^{(0)}(\epsilon) \equiv 1$. Similarly, for the solution of the adjoint eigenproblem,

$$
\psi_{0}(u)=\lambda_{0} \int_{-\infty}^{\infty} d \epsilon \psi_{0}(\epsilon) K(\epsilon, u)
$$

we write

$$
\psi_{0}(\epsilon)=\psi_{0}^{(0)}(\epsilon)+\alpha \psi_{0}^{(1)}(\epsilon)+O\left(\alpha^{2}\right),
$$

where $\psi_{0}^{(0)}(\epsilon)$ is the function that solves Eq. (10). Although we do not know this function explicitly, we have ascertained its existence in Sec. 3.1.

Inserting these series into Eq. (13), multiplying with the function $\psi_{0}(\epsilon)$, and comparing coefficients yields

$$
\begin{aligned}
\lambda_{0}= & 1+\alpha \int_{-\infty}^{\infty} d \epsilon \psi_{0}^{(0)}(\epsilon)\left[\Gamma_{1}(\epsilon) / \Gamma_{0}(\epsilon)\right] / \int_{-\infty}^{\infty} d \epsilon \psi_{0}^{(0)}(\epsilon) \\
& +O\left(\alpha^{2}\right) .
\end{aligned}
$$


Here we have defined

$$
\Gamma_{1}(\epsilon)=\Gamma_{0}(\epsilon) \int_{-\infty}^{\infty} d u K_{1}(\epsilon, u) .
$$

We see that the first order correction to the eigenvalue $\lambda_{0}$ is given by an average of the rate ratio $\Gamma_{1} / \Gamma_{0}$ with the zeroth order left eigenfunction $\psi_{0}^{(0)}$ as the weight:

$$
\lambda_{0}=1+\alpha\left\langle\Gamma_{1} / \Gamma_{0}\right\rangle_{\psi_{0}^{(0)}},
$$

or

$$
\lambda_{0}^{(1)}=\left\langle\Gamma_{1} / \Gamma_{0}\right\rangle_{\psi_{0}^{(0)}},
$$

where $\langle f\rangle_{\psi}=\int_{-\infty}^{\infty} d \epsilon f(\epsilon) \psi(\epsilon) / \int_{-\infty}^{\infty} d \epsilon \psi(\epsilon)$.

\subsection{A perturbative solution of the full integral equation}

The desired solution of Eq. (13) can now be written in terms of a resolvent $R(\epsilon, u)$, see ( $\mathrm{S} 46)$,

$$
\varphi(\epsilon)=\frac{1}{\Gamma_{0}(\epsilon)}+\int_{-\infty}^{\infty} d u R(\epsilon, u) \frac{1}{\Gamma_{0}(u)},
$$

and the resolvent can be expanded in a Laurent series (S Sec.8),

$$
R(\epsilon, u)=\frac{a_{-1}(\epsilon, u)}{1-\lambda_{0}}+O\left(\left(1-\lambda_{0}\right)^{0}\right) .
$$

Since $1-\lambda_{0}=O(\alpha)$, keeping only the leading term in the Laurent series is consistent with working to leading order in $\alpha$. The residue $a_{-1}$ satisfies the integral equation (S Sec.8)

$$
\begin{aligned}
a_{-1}(\epsilon, u) & =\lambda_{0} \int_{-\infty}^{\infty} d t K(\epsilon, t) a_{-1}(t, u) \\
& =\int_{-\infty}^{\infty} d t K_{0}(\epsilon, t) a_{-1}(t, u)+O(\alpha) .
\end{aligned}
$$

If we define

$$
A_{-1}(\epsilon)=\int_{-\infty}^{\infty} d u a_{-1}(\epsilon, u) / \Gamma_{0}(u)
$$

we have

$$
A_{-1}(\epsilon)=\int_{-\infty}^{\infty} d t K_{0}(\epsilon, t) A_{-1}(t)+O(\alpha) .
$$

To lowest order in $\alpha$, this is just Eq. (8) with $\lambda=1$, which has a solution

$$
A_{-1}(\epsilon)=\text { const. }+O(\alpha) \equiv A_{-1}+O(\alpha) .
$$

The solution of the integral equation can now be written

$$
\varphi(\epsilon)=\frac{A_{-1}}{\alpha} \frac{1}{\lambda_{0}^{(1)}}+O\left(\alpha^{0}\right) .
$$

We see that to leading order in the Laurent expansion in $\alpha, \varphi$ does not depend on $\epsilon$. We also see that the solution does not exist for $\alpha=0$, as we had anticipated in Sec. 3.2 above.

The remaining task is to determine the constant $A_{-1}$, which is not fixed by the integral equation (21b). Let us consider the functions $\varphi(\epsilon)$ and $1 / \Gamma_{0}(\epsilon)$ as elements $|\varphi\rangle$ and $\left|1 / \Gamma_{0}\right\rangle$, respectively, of a linear space, and the kernel $K$ a linear operator in that space. The integral equation (13) then reads

$$
|\varphi\rangle=\left|1 / \Gamma_{0}\right\rangle+K|\varphi\rangle .
$$

Multiplying from the left with the left eigenfunction $\left\langle\psi_{0}\right|$ of $K$, we have

$$
\begin{aligned}
\left\langle\psi_{0} \mid \varphi\right\rangle & =\left\langle\psi_{0} \mid 1 / \Gamma_{0}\right\rangle+\left\langle\psi_{0}|K| \varphi\right\rangle \\
& =\left\langle\psi_{0} \mid 1 / \Gamma_{0}\right\rangle+\lambda_{0}\left\langle\psi_{0} \mid \varphi\right\rangle .
\end{aligned}
$$

From Eq. (22) we know that, to lowest order in $\alpha,|\varphi\rangle$ is a multiple of $\left|\varphi_{0}^{(0)}\right\rangle$,

$$
|\varphi\rangle=\frac{1}{\alpha} \frac{A_{-1}}{\lambda_{0}^{(1)}}\left|\varphi_{0}^{(0)}\right\rangle+O\left(\alpha^{0}\right) .
$$

To leading order in $\alpha$ we thus have

$$
\left(1-\lambda_{0}\right)\left\langle\psi_{0}^{(0)} \mid \phi_{0}^{(0)}\right\rangle[1+O(\alpha)]=\left\langle\psi_{0}^{(0)} \mid 1 / \Gamma_{0}\right\rangle,
$$

which yields

$$
A_{-1}=\frac{-\left\langle\psi_{0}^{(0)} \mid 1 / \Gamma_{0}\right\rangle}{\left\langle\psi_{0}^{(0)} \mid \varphi_{0}^{(0)}\right\rangle} .
$$

Using the notation defined in Eqs. (17) we have

$$
\varphi(\epsilon)=\frac{1}{\alpha} \frac{\left\langle 1 / \Gamma_{0}\right\rangle_{\psi_{0}^{(0)}}}{\left\langle\Gamma_{1} / \Gamma_{0}\right\rangle_{\psi_{0}^{(0)}}}+O\left(\alpha^{0}\right) .
$$

Finally, using the observation made after Eq. (13) we can put $\alpha=1$ and write

$$
\varphi(\epsilon)=\frac{\left\langle 1 / \Gamma_{0}\right\rangle_{\psi_{0}^{(0)}}}{\left\langle\Gamma_{1} / \Gamma_{0}\right\rangle_{\psi_{0}^{(0)}}}+O\left(\Gamma_{1}^{0}\right) .
$$

This is the desired solution of the integral equation (7a) to leading order in the function $\Gamma_{1}(\epsilon)$ defined in Eq. (17b). The only assumption we have made is that the left eigenfunction $\psi_{0}^{(0)}(\epsilon)$, which we have not explicitly determined, falls off sufficiently fast for large $\epsilon$ to ensure the existence of the averages in Eq. (27).

The above result is exact. Let us compare it with the popular approximation [4] that replaces $\varphi(u)$ under the integral in Eq. (7a) by $\varphi(\epsilon)$. This turns the integral equation into an algebraic one, and the solution is

$$
\varphi(\epsilon) \approx 1 / \Gamma_{1}(\epsilon)
$$


This has the same structure in terms of $\Gamma_{1}$ as the exact solution (28), and leads to the same temperature dependence of the conductivity, as we will see. However, it is uncontrolled and cannot be justified other than by a comparison with the exact solution provided above, or with a solution of the Boltzmann equation.

\section{The electrical conductivity}

\subsection{The Drude formula}

We now can write the conductivity as follows. From Eq. (1) we have

$$
\sigma=\frac{n_{\mathrm{e}} e^{2}}{2 m_{\mathrm{e}}} \int_{-\infty}^{\infty} \frac{d \epsilon}{4 T} \frac{1}{\cosh ^{2}(\epsilon / 2 T)} \frac{1}{\Gamma_{\operatorname{tr}}(\epsilon)} .
$$

Equation (29) shows that, to lowest order in $\Gamma_{1}, \Gamma_{\text {tr }}$ is independent of $\epsilon$ and given by

$$
\Gamma_{\mathrm{tr}}=\frac{\left\langle\Gamma_{1} / \Gamma_{0}\right\rangle_{\psi_{0}^{(0)}}}{\left\langle 1 / \Gamma_{0}\right\rangle_{\psi_{0}^{(0)}}}+O\left(\Gamma_{1}^{2}\right) .
$$

We thus have as our final result the Drude formula

$$
\sigma=\frac{n_{\mathrm{e}} e^{2} \tau_{\mathrm{tr}}}{m_{\mathrm{e}}}
$$

with a transport relaxation rate

$$
\frac{1}{\tau_{\mathrm{tr}}}=2 \Gamma_{\mathrm{tr}}
$$

Here $\Gamma_{\text {tr }}$ is given by Eq. (32). It depends on $\Gamma_{0}$ from Eq. (3), $\Gamma_{1}$ from Eq. (17b), the kernels $K_{0}$ and $K_{1}$ defined by Eq. (6), and the left eigenfunction $\psi_{0}^{(0)}$ of $K_{0}$ that is defined as the solution of the equation

$$
\psi_{0}^{(0)}(u)=\int_{-\infty}^{\infty} d \epsilon \psi_{0}^{(0)}(\epsilon) K_{0}(\epsilon, u) .
$$

\subsection{Two physical examples}

We finally illustrate our result with two examples. One is the well-known result for the conductivity due to electron-phonon scattering, the other, the conductivity due to the scattering of helical magnon excitations in helimagnets. In both cases, the effective potentials $\bar{V}_{0,1}^{\prime \prime}$ that determine the kernels have the following powerlaw form in the limit of low frequencies:

$$
\bar{V}_{m}^{\prime \prime}(u)=\frac{-C_{m}}{N_{\mathrm{F}}}|u|^{n_{m}-1} \operatorname{sgn} u, \quad(m=0,1),
$$

where $C_{0}$ and $C_{1}$ are positive constants. For acoustic phonons, the exponents $n_{m}$ are [7]

$$
n_{0}=3, \quad n_{1}=5 \quad \text { (phonons). }
$$

For helimagnons, the corresponding values are [5]

$$
n_{0}=3 / 2, \quad n_{1}=5 / 2 \quad \text { (helimagnons). }
$$

For the relaxation rates $\Gamma_{0,1}$ this yields

$$
\Gamma_{m}(\epsilon)=T^{n_{m}} \gamma_{m}(\epsilon / T), \quad(m=0,1),
$$

where

$$
\begin{array}{r}
\gamma_{m}(x)=C_{m} \int_{0}^{\infty} d u u^{n_{m}-1}[2 n(u)+f(u+x) \\
+f(u-x)] .
\end{array}
$$

Finally, it follows from Eqs. (6) and (33c) that $\psi_{0}^{(0)}(\epsilon)$ is a function of $\epsilon / T$ only. For the transport relaxation time, and hence for the conductivity, we thus have the a temperature dependence from electron-phonon scattering:

$$
\sigma \propto \tau_{\operatorname{tr}} \propto 1 / T^{5} \quad \text { (phonons) }
$$

This is the well-known Bloch-Grüneisen law [1]. The same power law is obtained by means of the uncontrolled approximation (30) [4], but the prefactor is different. For scattering from helimagnons we find

$$
\sigma \propto \tau_{\operatorname{tr}} \propto 1 / T^{5 / 2} \quad \text { (helimagnons). }
$$

Again, the uncontrolled approximation yields the same $1 / T^{5 / 2}$ temperature behavior [5]. The above considerations ascertain that this is indeed the exact lowtemperature contribution to the electrical conductivity due to electron-helimagnon scattering.

\section{Conclusion}

In conclusion, we have considered the Kubo formula for the electrical conductivity due to the scattering of electrons by bosonic excitations. We have given an exact solution of the integral equation for the vertex function that determines the transport relaxation rate. This exact solution has the qualitatively same behavior as the result of a popular, but uncontrolled, approximation. For the scattering of electrons by acoustic phonons this yields the usual Bloch-Grüneisen law for the electrical resistivity, $\rho \propto T^{5}$, and for the helimagnon contribution to the conductivity in helical 
magnets it confirms the result $\rho \propto T^{5 / 2}$ that had been previously obtained by means of solving the integral equation approximately. All of these results are consistent with what is known from solutions of the Boltzmann equation.

\section{Acknowledgments}

We thank N. Christopher Phillips for a helpful discussion. This work was supported by the NSF under grant Nos. DMR-05-30314 and DMR-05-29966.

\section{References}

[1] See, e.g., A.H. Wilson, The Theory of Metals, Cambridge University Press, Cambridge 1954

[2] R. Kubo, J. Phys. Soc. Japan 12 (1957) 570.

[3] Holstein has shown that the Kubo approach is equivalent to a set of two linearized kinetic equations of Boltzmann type: T. Holstein, Ann. Phys. (N.Y.) 29, (1964) 410.

[4] G.D. Mahan, Many-Particle Physics, Plenum, New York 1981.

[5] D. Belitz, T.R. Kirkpatrick, and A. Rosch, Phys. Rev. B 74 (2006 124409 [Erratum: Phys. Rev. B 76 (2007) 149902]; T.R. Kirkpatrick, D. Belitz, and R. Saha, Phys. Rev. B 78 (2008) 094408.

[6] V.I. Smirnov, A Course of Higher Mathematics, Translated by D.E. Brown, Translation edited by I.N. Sneddon, Pergamon, Oxford 1964, Chapter 1.

[7] See, e.g., A.A. Abrikosov, L.P. Gorkov, and I.E. Dzyaloshinski, Methods of Quantum Field Theory in Statistical Physics, Dover, New York 1963. 